\title{
Boundary-based corner detection using eigenvalues of covariance matrices
}

\author{
D. M. Tsai and H. T. Hou and H. J. Su \\ Machine Vision Lab. \\ Department of Industrial Engineering and Management \\ Yuan-Ze University, Chung-Li, Taiwan, R.O.C. \\ E-mail: iedmtsai@ saturn.yzu.edu.tw
}

\begin{abstract}
In this paper we present a new measure for corner detection based on the eigenvalues of the covariance matrix of boundary points over a small region of support. It avoids false alarms for superfluous corners on circular arcs. Experimental results have shown that the proposed corner detection methods using curvature measures. It has good detection and localization for curved objects in different rotations and with varying scale changes.
\end{abstract}

Keywords. Corner detection; Covariance matrices; Eigenvalues; Curvature 


\section{Introduction}

Corner detection in digital images has been shown to be extremely useful in many computer vision applications. Since information about a shape is concentrated at the corners, they prove to be practical descriptive primitives in shape representation, objection recognition (Han and Jang, 1990) and motion analysis (Costabile el al., 1985).

Corner detection techniques can be classified into two major categories: boundary-based approaches and gray-level approaches. Boundary-based approaches detect corners on the boundaries of objects. Gray-level approaches directly work on gray-level images by matching corner templates (Mehrotra and Nichani, 1990) or by computing gradients at edge points (Singh and Shneier, 1990; Rosin, 1996). The proposed method in this paper is a boundary-based approach.

Corners on a curve arise where two relatively straight-line segments intersect. The detection procedure for corners on object boundaries involves, first, segmenting a scene image into meaningful regions, and then extracting boundaries from the regions of interest. Corners on the extracted boundaries are typically identified for those points with high curvature. Methods of discrete curvature measures are based on the rate of change of tangent direction. The tangent angle is generally measured by calculating the first difference of direction change over a small curve segment, and the curvature measurement is based on the second order difference that calculates the change of tangential direction over the curve segment.

Many discrete algorithms have been developed to compute the curvature of 
boundary points lying on a digital curve (Worring and Smeulders, 1993; Fairney and Fairney, 1994; Tsai and Chen, 1994). Tsai (1997) measures the curvature by using neural networks to recognize the included angles at boundary points. Sohn et al. (1998) propose a method of boundary smoothing for curvature estimation using a deterministic approximation of simulated annealing. A variety of curvature measurement methods have also been reported by Teh and Chin (1989). The existing curvature-based corner detection methods (Beus and Tiu, 1987; Anderson and Bezdek, 1984; Liu and Srinath, 1990) generally work reliably for polygonal objects since the vertices of polygons have large curvature values and the points elsewhere on the boundary have approximately zero curvature. However, they may detect many spurious corners for the objects involving circular arcs of varying radii. Points on the circular arc of a small radius generally have high curvature, and may have curvature values large than the intersection point of two stright-line segments in the discrete domain. Two or more superfluous corners detected on a digital circular arc is not uncommon using the curvature measures. The detection and localization become poor and unstable when the objects of curved shapes can be changed in scales and rotated in arbitrary orientations.

Instead of using the curvature measures for corner detection, in this paper we propose a new corner detection method based on the eigenvalues of the covariance matrix of data points on a curve segment. The goal of the proposed corner detector is to possess robust detection for object shapes containing various curved and circular arcs.

This paper is organized as follows. Section 2 introduces the covariance matrix of a curve segment, and then discusses the use of the eigenvalue of the matrix as a 
quantitative measure of corners. Section 3 presents the experimental results. The conclusion is reached in section 4.

\section{Covariance matrices and their eigenvalues}

In this paper, the measure for the prominence of a corner is derived from the statistical and geometric properties associated with the eigenvalues of the covariance matrix of data points on a digital boundary over a region of support.

Let the sequence of $\mathrm{n}$ digital points describe the boundary $\mathrm{P}$ of an object,

$$
\mathrm{P}=\left\{p_{i}=\left(x_{i}, y_{i}\right), i=1,2, \uparrow, n\right\}
$$

where $p_{i+1}$ is a neighbor of $p_{i}$ (modulo $\left.n\right)$, and $\left(x_{i}, y_{i}\right)$ are the Cartesian coordinates of $p_{i}$ in the image. Denote $S_{k}\left(p_{i}\right)$ as a small curve segment of $\mathrm{P}$, which is defined by the region of support between points $p_{i-k}$ and $p_{i+k}$ for some integer $k$, i.e.

$$
S_{k}\left(p_{i}\right)=\left\{p_{j} \mid j=i-k, i-k+1, \uparrow, i+k-1, i+k\right\}
$$

The covariance matrix $C$ of a curve segment $S_{k}\left(p_{i}\right)$ is given by

$$
C=\left[\begin{array}{ll}
c_{11} & c_{12} \\
c_{21} & c_{22}
\end{array}\right]
$$


where

$$
\begin{aligned}
& c_{11}=\left[\frac{1}{2 k+1} \sum_{j=i-k}^{i+k} x_{j}^{2}\right]-c_{x}^{2} \\
& \left.\left.c_{12}=c_{21}=\left[\frac{1}{2 k+1} \sum_{j=i-k}^{i+k} x_{j}\right] y_{j}\right]-c\right] c_{y} \\
& c_{22}=\left[\frac{1}{2 k+1} \sum_{j=i-k}^{i+k} y_{j}^{2}\right]-c_{y}^{2}
\end{aligned}
$$

$c_{x}$ and $c_{y}$ are the geometrical center of the curve segment $S_{k}\left(p_{i}\right)$, i.e.

$$
\begin{aligned}
& c_{x}=\frac{1}{2 k+1} \sum_{j=i-k}^{i+k} x_{j} \\
& c_{y}=\frac{1}{2 k+1} \sum_{j=i-k}^{i+k} y_{j}
\end{aligned}
$$

The covariance matrix is $2 \times 2$, symmetric and positive semidefinite. There are two eigenvalues $\lambda_{L}$ and $\lambda_{S}$ for the matrix $C$, which are

$$
\begin{aligned}
& \lambda_{L}=\frac{1}{2}\left[c_{11}+c_{22}+\sqrt{\left(c_{11}-c_{22}\right)^{2}+4 c_{12}^{2}}\right] \\
& \lambda_{S}=\frac{1}{2}\left[c_{11}+c_{22}-\sqrt{\left(c_{11}-c_{22}\right)^{2}+4 c_{12}^{2}}\right]
\end{aligned}
$$

The eigenvalues of the matrix $C$ can be used to extract the shape information about a curve. It can be shown that when the shape $S$ is a stright-line segment, the smaller eigenvalue $\lambda_{s}$ for the line segment in the continuous domain will be zero, regardless of the length and orientation of the line segment. If the shape $S$ is an ellipse, then $\lambda_{L}>\lambda_{S}$ and $\sqrt{\lambda_{L}}$ and $\sqrt{\lambda_{S}}$ are the semimajor and semiminor axial lengths of the ellipse. The two eigenvalues will be equal if the shape $S$ is a full 
circle. Therefore, the smaller eigenvalue $\lambda_{s}$ of the covariance matrix $C$ can be utilized to measure the prominence of a corner for each boundary point $p_{i}$ over the curve segment $S_{k}\left(p_{i}\right)$. A point $p_{i}$ is said to be a corner if its $\lambda_{S}$ value exceeds a predetermined threshold, and individual corners are separated by a spacing of at least $k$ points. Points on a straight-line or on a flat curve segment will result in small $\lambda_{S}$ values approximate to zero, whereas points on sharp corners will generate large $\lambda_{S}$ values. To verify the usefulness of the eigenvalue $\lambda_{S}$ for corner detection in discrete images, the following three analytic curves are analyzed:

1) A straight-line $y=\left(\tan ^{-1} \theta\right) x$, where $\theta$ is the slope angle of the line.

2) A circle $x^{2}+y^{2}=r^{2}$, where $\mathrm{r}$ is the radius of the circle.

3) An angle defined by two intersecting lines

$$
y=\left[\tan ^{-1}\left(90^{\circ}-\frac{\varphi}{2}\right)\right]|x|,
$$

where $\varphi$ is the included angle of the two intersecting lines.

The three analytic curves as shown in Figure 1 are generated in an image of size $512 \times 480$ pixels. The digital curves are created such that

$$
\max \left\{\left|x_{i}-x_{i-1}\right|,\left|y_{i}-y_{i-1}\right|\right\}=1, \forall i
$$

where $\left(x_{i}, y_{i}\right)$ are generated from the analytic equations and truncated to their 
nearest integers. They represent the grid coordinates of the discrete image.

Tables $1(\mathrm{a}), 1(b)$ and $1(c)$ summarize the eigenvalues $\lambda_{s}$ for the three analytic curves under varying parameter setups. Two different regions of support $k=10$ and $k=15$, which correspond to 21 and 31 data points, respectively, for each curve segment, are examined in the experiments. Note that the eigenvalues $\lambda_{L}$ and $\lambda_{s}$ are associated with the major and minor axial lengths of the ellipse, the size of region of support (i.e., the number of data points used to construct the covariance matrix) will affect the eigenvalues of a given boundary. It can be seen from Table 1(a) that the values of $\lambda_{s}$ are approximate to zero (less than $10^{-5}$ ) regardless of the line orientations and lengths. Typically, one selects the region of support $k$ in the rang between 5 and 15 . The region of support for the computation of the covariance matrix at each boundary point can also be adaptively selected. Teh and Chin (1989) have proposed an effective approach for determining the region of support at each individual boundary point based on local properties of the point.

The measure of curvature is equivalent to the reciprocal of the radius of an arc. Therefore, a smaller circle will result in larger curvature. The trend is also consistently revealed in Table $1(b)$. It shows that the increment of the radius yields the decrement of the $\lambda_{s}$ value. All $\lambda_{s}$ eigenvalues of circular arcs are relatively larger than those of straight-line segments. It can also be observed from Table $1(c)$ that a sharper angle (smaller included angle) results in larger $\lambda_{s}$ value. The $\lambda_{s}$ values of sharp angles are significantly larger than those found on circular arcs. This huge gap of $\lambda_{s}$ values between sharp angles and circular arcs helps to eliminate the false detection of superfluous corners on circular arcs. In summary, a sharp angle has larger $\lambda_{s}$ value than the points on circular arcs. A point on the circular arc has larger $\lambda_{S}$ value than 
the points lying on straight lines. A point on the straight line has a $\lambda_{S}$ value approximate to zero. The sharper the angle or the smaller the radius of a circular arc is given, the larger the $\lambda_{s}$ value will be produced. Therefore, the eigenvalue $\lambda_{s}$ is a robust measure for corner detection.

\section{Experimental results}

In this section we present the experimental results to evaluate the performance of the proposed corner detector for artificial curved objects and real objects. All algorithms are programmed in $\mathrm{C}$ and executed on a personal computer. The image size is $512 \times 480$ in pixels. Based on the study of Liu and Srinath (1990), the Freeman-Davis-based algorithms (Freeman and Davis, 1977; Beus and Tiu, 1987) outperform other boundary-based corner detection schemes. Therefore, the performance of the proposed corner detector is compared with that of the Freeman-Davis method (Freeman and Davis, 1977). In the Freeman-Davis method, a corner is defined as an isolated discontinuity (local curvature) in the mean slope, its prominence being proportional to the length of the discontinuity-free regions to either side of the point as well as the measured magnitude of the discontinuity.

The thresholds for both the proposed corner detector and the Freeman-Davis method are selected, individually, so that the number of spurious corners are minimized while all desired corners specified by a human viewer are detected. The number of spurious corners, therefore, gives the performance criterion.

Let $C=\left\{c_{1}, c_{2}, \mathrm{i}, c_{m}\right\}$ be a set of $\mathrm{m}$ desired corners specified by a human 
viewer, and $\lambda_{S}\left(c_{i}\right)$ the measured $\lambda_{S}$ value at corner $c_{i}$. The threshold $T_{\lambda_{S}}$ is selected such that

$$
T_{\lambda_{s}}=\min \left\{\lambda_{s}\left(c_{i}\right), i=1,2, \uparrow, m\right\}
$$

The region of support $k$ for calculating the covariance matrix is 10 throughout all experiments for both methods so that their performances can be compared in the same basis.

Three curved objects with their desired corners specified by a human viewer are shown in Figures 2(a), 2(b) and 2(c). These three curved objects have been tested under three different scales $50 \%, 75 \%$ and $100 \%$ in terms of area changes, and objects of scale $100 \%$ are rotated in two additional orientations of $30^{\circ}$ and $60^{\circ}$ so that the effects of scale and orientation on detected corners can be analyzed. Figures 3, 4 and 5 show the detection results of the Freeman-Davis method (figures in the left column). Every detected corner in the figures is marked by a cross " + " for the proposed method, and by a circle " $\bigcirc$ " for the Freeman-Davis method. Table 2 presents the number of spurious corners detected by the two methods for the three curved objects in different scales and orientations. It reveals that the Freeman-Davis method detects a few spurious corners on arc segments, whereas the proposed method identifies all desired corners without any false alarm. The proposed corner detector is robust and reliable for curved objects under varying scales and orientations.

In terms of localization, the distance (in pixels) between a detected corner point and its corresponding true corner for the three curved objects under three scaling factors $50 \%, 75 \%$ and $100 \%$ and two rotational angles $30^{\circ}$ and $60^{\circ}$ is within 1 pixel using the proposed corner detector. 
Three real objects are also used in experiments to demonstrate the performance of the proposed $\lambda_{S}$ measure. Figure 6(a) presents a natural leaf. The backlighting technique is used to obtain the silhouette of the leaf, as shown in Figure 6(b). The corner detection result is shown in Figure 6(c). Figure 7(a) presents the silhouette of a gear with 20 teeth. Figure 7(b) shows that the corners of each tooth are reliably detected in all directions. Figures 8(a) and 8(b) show the profile of a Mackerel shark (Saund, 1992) and the corner detection result, respectively. Figure 8(c) illustrates a distorted version of the shark. Figure 8(d) shows that all desired corners as indicated in Figure 8(b) are also reliably detected from the noisy boundary of the shark.

Finally, the proposed method is also compared with a novel scale-space corner detection scheme developed by Rattarangsi and Chin (1992). The technique is based on a Gaussian scale-space, which consists of the maxima of absolute curvature of the boundary function presented at all scales. Figure 9(a) shows a key-shape example used in their study. From the detection results we find that four spurious corners on the circular arc are detected using the scale-space corner detection method (Figure 9(b)), and none of the boundary points on the arc are selected as corner points using the proposed method (Figure 9(c)).

\section{Conclusions}

Instead of using the conventional curvature measures that evaluate the tangent change along the object boundary for corner detection, in this paper we have presented a new quantitative measure of corners based on the smaller eigenvalue of the covariance matrix of boundary points over a small region of support. The 
eigenvalue associated with the minor axis increases its magnitude as a point on the curve segment increases its curvature. It has better discrimination between true corners and spurious corner points on circular arcs.

The proposed corner detector is computationally fast and easy to implement. More importantly, it avoids false alarms for superfluous corners on circular arcs. The experiments aforementioned have shown that the proposed corner detector compares flavorably with the Freeman-Davis method. It has generated good detection and localization for curved objects under different rotation and scale changes. 
Table 1. The eigenvalues $\lambda_{s}$ for three analytic curves under different parameter values and regions of support.

(a) A line $y=\tan ^{-1} \theta$

\begin{tabular}{|c|c|c|}
\hline \multirow{2}{*}{ Slope angle $\theta$} & \multicolumn{2}{|c|}{ Region of support } \\
\cline { 2 - 3 } & 21 data points & 31 data points \\
\hline $0^{0}$ & $-1.907 \mathrm{E}-06$ & $7.529-\mathrm{E}-06$ \\
\hline $20^{0}$ & $3.814 \mathrm{E}-06$ & $-9.536 \mathrm{E}-05$ \\
\hline $40^{\circ}$ & $-7.629 \mathrm{E}-06$ & $3.433 \mathrm{E}-05$ \\
\hline $60^{\circ}$ & $3.814 \mathrm{E}-06$ & $3.814 \mathrm{E}-05$ \\
\hline $80^{\circ}$ & $-2.670 \mathrm{E}-05$ & $3.051 \mathrm{E}-05$ \\
\hline
\end{tabular}

(b) A circle $x^{2}+y^{2}=r^{2}$

\begin{tabular}{|c|c|c|}
\hline \multirow{2}{*}{ Radius r (pixels) } & \multicolumn{2}{|c|}{ Region of support } \\
\cline { 2 - 3 } & 21 data points & 31 data points \\
\hline 30 & 0.256 & 1.397 \\
\hline 50 & 0.090 & 0.465 \\
\hline 70 & 0.043 & 0.233 \\
\hline 90 & 0.024 & 0.141 \\
\hline
\end{tabular}

(c) An angle $y=\left[\tan ^{-1}\left(90^{\circ}-\frac{\varphi}{2}\right)\right]|x|$

\begin{tabular}{|c|c|c|}
\hline \multirow{2}{*}{ Included angle $\varphi$} & \multicolumn{2}{|c|}{ Region of support } \\
\cline { 2 - 3 } & 21 data points & 31 data points \\
\hline $30^{0}$ & 6.969 & 15.756 \\
\hline $50^{0}$ & 5.952 & 13.448 \\
\hline $70^{0}$ & 4.845 & 10.940 \\
\hline $90^{0}$ & 3.673 & 8.291 \\
\hline
\end{tabular}

Table 2. The number of spurious corners detected by the Freeman-Davis method and the propoosed method.

\begin{tabular}{|c|c|c|c|c|c|c|c|}
\hline \multicolumn{2}{|c|}{ Object } & \multicolumn{2}{c|}{$\begin{array}{c}\text { Test object 1 } \\
\text { (Figure 3) }\end{array}$} & \multicolumn{2}{c|}{$\begin{array}{c}\text { Test object 2 } \\
\text { (Figure 4) }\end{array}$} & \multicolumn{2}{c|}{$\begin{array}{c}\text { Test object 3 } \\
\text { (Figure 5) }\end{array}$} \\
\hline \multicolumn{2}{|c|}{ Method } & Freeman-Davis & Proposed & Freeman-Davis & Proposed & Freeman-Davis & Proposed \\
\hline \multirow{3}{*}{ Scale } & $50 \%$ & 2 & 0 & 8 & 0 & 5 & 0 \\
\cline { 2 - 8 } & $75 \%$ & 2 & 0 & 12 & 0 & 4 & 0 \\
\cline { 2 - 8 } & $100 \%$ & 2 & 0 & 7 & 0 & 8 & 0 \\
\hline \multirow{2}{*}{ Rotation } & $30^{\circ}$ & 4 & 0 & 3 & 0 & 4 & 0 \\
\cline { 2 - 8 } & $60^{\circ}$ & 1 & 0 & 4 & 0 & 4 & 0 \\
\hline
\end{tabular}




\section{References}

Anderson, I. M. and J. C. Bezdek (1984). Curvature and tangential deflection of discrete arcs: a theory based on the commutator of scatter matrix pairs and its application to vertex detection in planar shape data. IEEE Trans. Pattern Analysis Mach. Intell. PAMI-6, 27-40.

Beus, H. L. and S. S. H. Tiu (1987). An improved corner detection algorithm based on chain-coded plane curves. Pattern Recognition 20, 291-296.

Costabile, M. F., C. Guerra and G. G. Pieroni (1985). Matching shapes: a case study in time varying images. Comput. Vision Graphic Image Process. 29, 296-310.

Fairney, D. P. and P. T. Fairney(1994). On the accuracy of point curvature estimators in a discrete environment. Image and Vision Comput. 12, 259-265.

Freeman, H. and L. S. Davis (1977). A corner-finding algorithm for chain-coded curves. IEEE Trans. Comput. C-26, 297-303.

Han, M. -H. and D. Jang (1990). The use of maximum curvature points for the recognition of partially occluded objects. Pattern Recognition 23, 21-33.

Liu, H. -C. and M. D. Srinath (1990). Corner detection from chain-code. Pattern Recognition 23, 51-68.

Mehrotra, R. and S. Nichani (1990). Corner detection. Pattern Recognition 23, 1223-1233.

Rattarangsi, A. and R. T. Chin (1992). Scale-based detection of corners of planar curves. IEEE Trans. Pattern Analysis Mach. Intell. PAMI-14, 430-449.

Rosin, P. L. (1996). Augmenting corner descriptors. Graphical Models and Image Processing 58, 286-294.

Saund, E. (1992). Putting knowledge into a visual shape representation. Artificial Intelligence 54, 71-119.

Singh, A. and M. Shneier (1990). Gray level corner detection a generalization and a robust real time implementation. Comput. Vision Graphics Image Process. 51,54-69. 
Sohn, K., J. H. kim and W. E. Alexander (1998). A mean field annealing approach to robust corner detection. IEEE Trans. Systems, Man, Cyber., Part B, 28, 82-90.

Teh, C. - H. and R. T. Chin (1989). On the detection of dominant points on digital curves. IEEE Trans. Pattern Analysis Mach. Intell. 11, 859-872.

Tsai, D. -M. (1997). Boundary-based corner detection using neural networks. Pattern Recognition 30, 85-97.

Tsai, D. -M. and M. -F. Chen (1994). Curve fitting approach for tangent angle and curvature measurements. Pattern Recognition 27, 699-711.

Worring, M. and A. W. M. Smeulders (1993). Digital curvature estimation. CVGIP: Image Understanding 58, 366-382. 


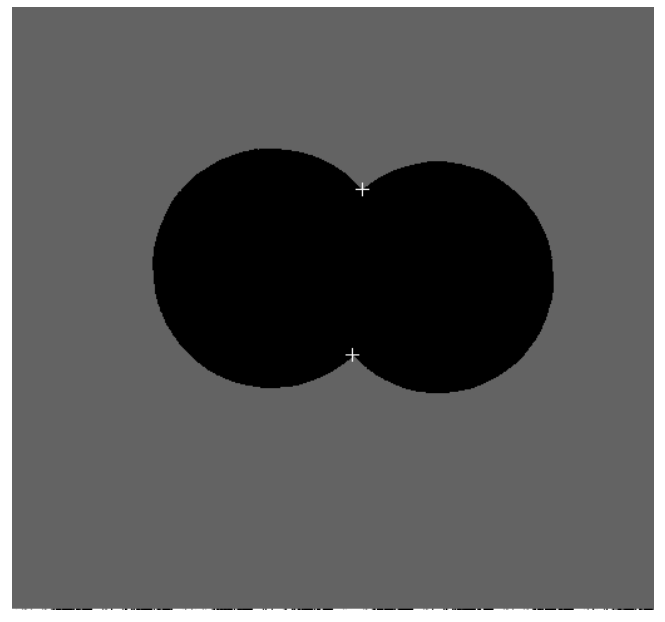

(a) Test object 1

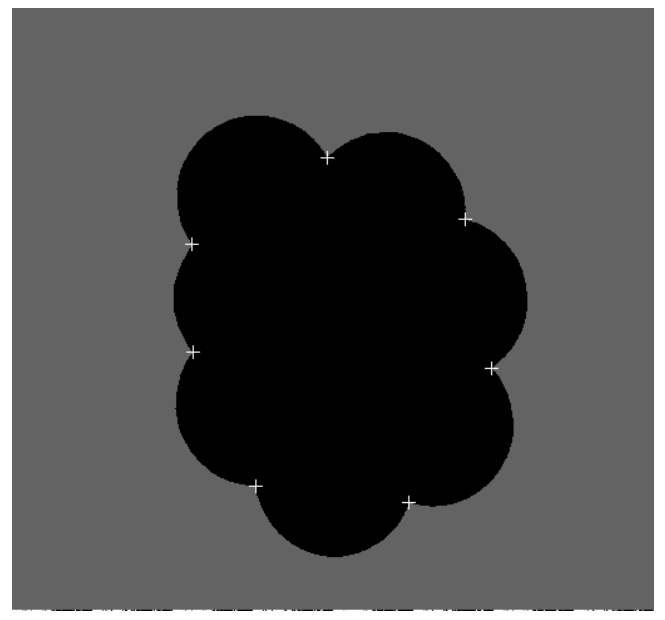

(b) Test object 2

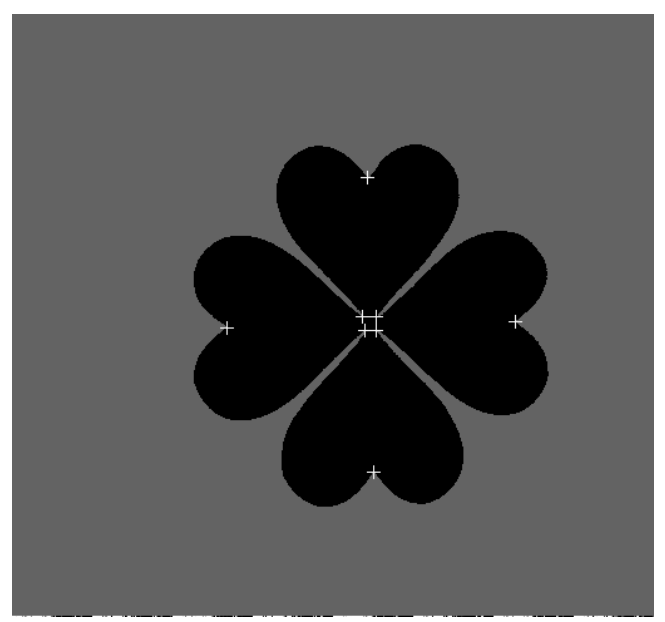

(c) Test object 3

Figure 2. Three real curved objects with their desired corners marked by "+ ". 

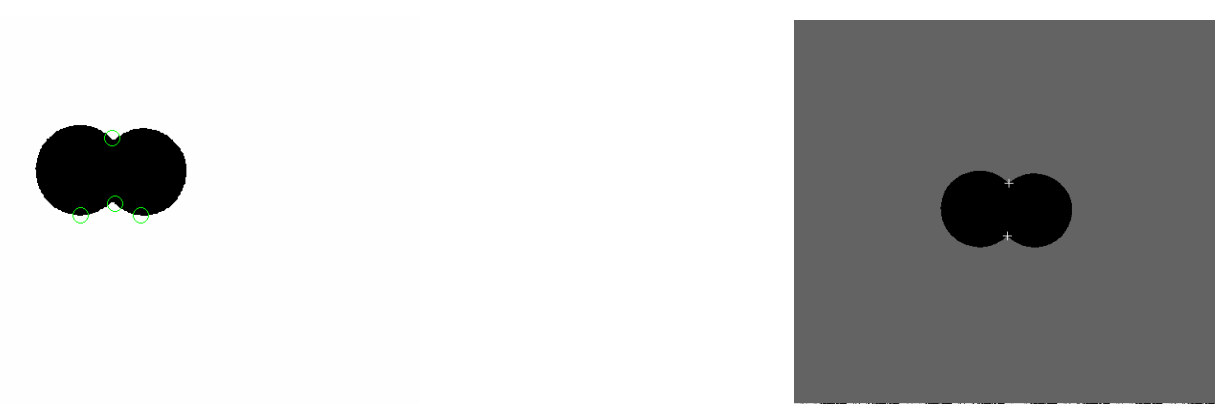

(a) Scaling factor $50 \%$
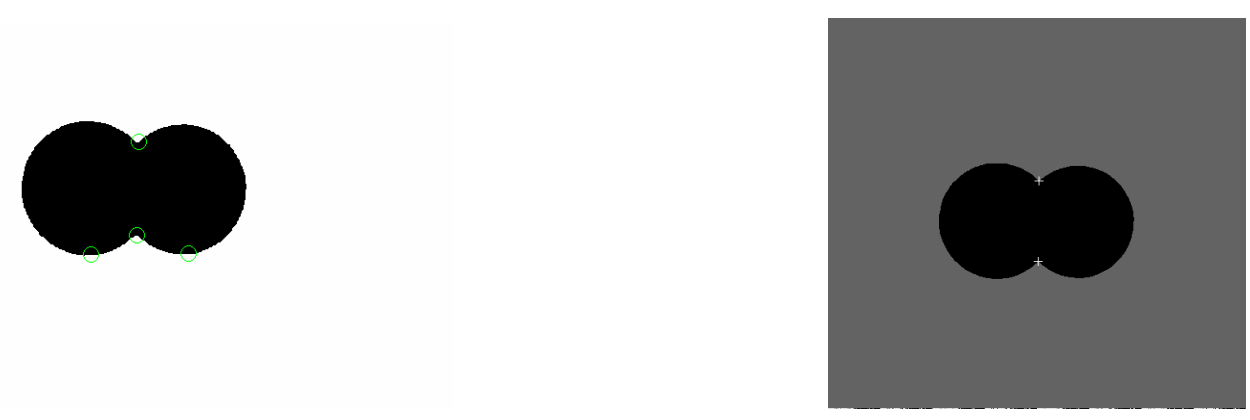

(b) Scaling factor $75 \%$
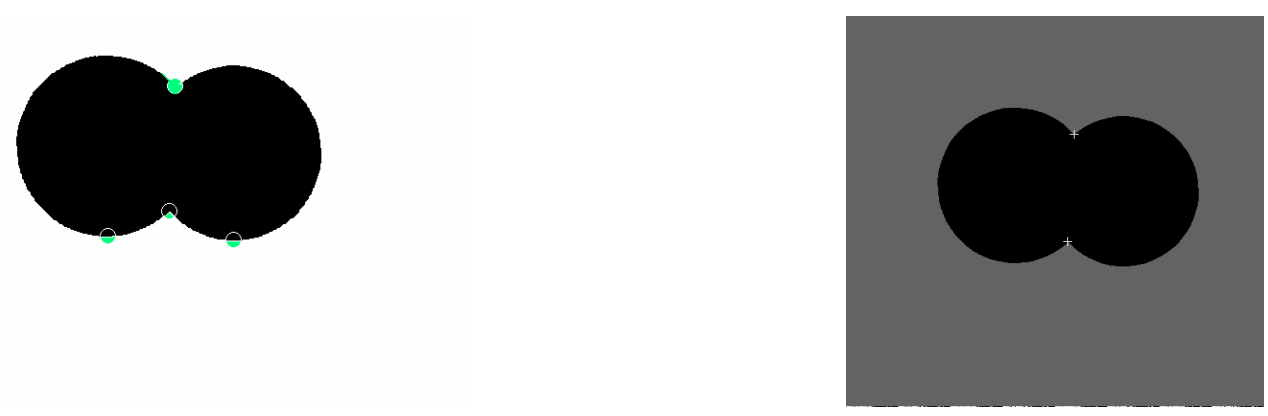

(c) Scaling factor $100 \%$
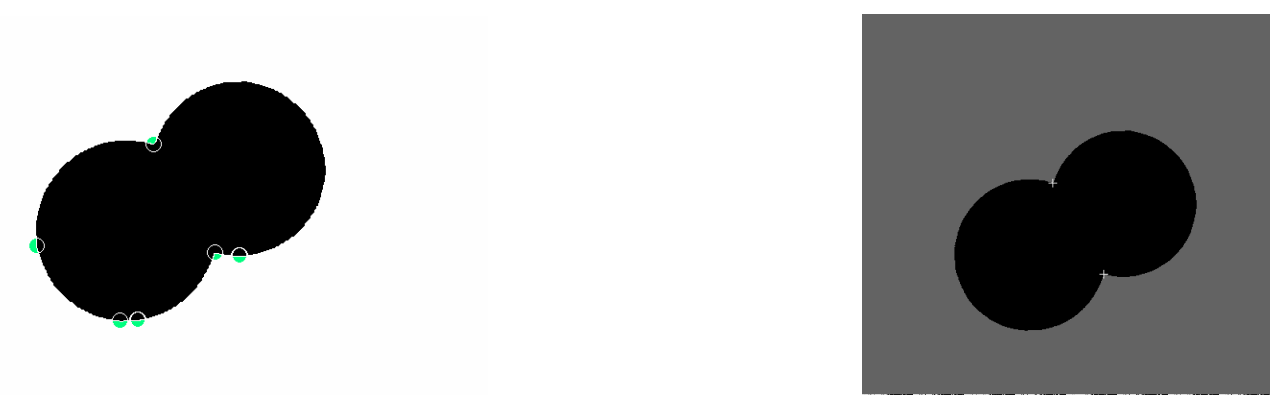

(d) Rotational angle $30^{\circ}$
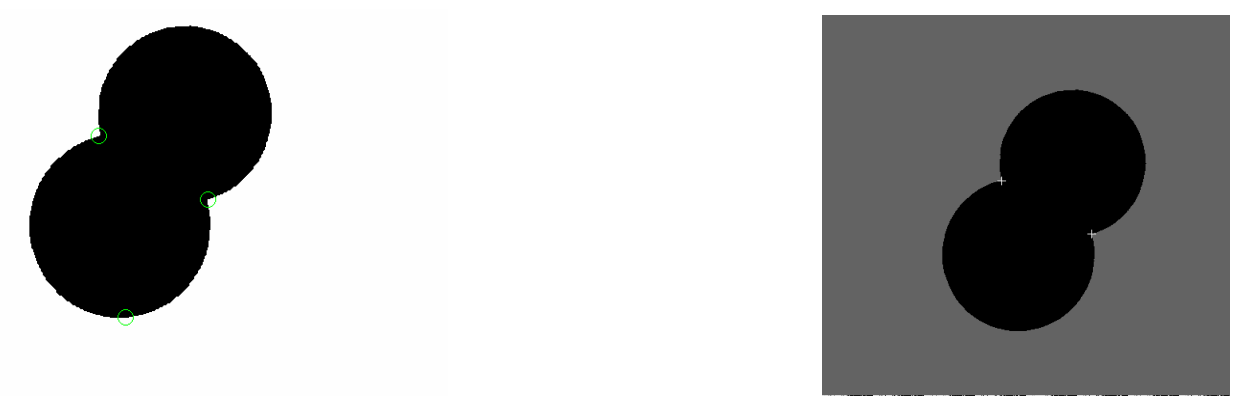
(d) Rotational angle $60^{\circ}$

Figure 3. Detected corners of test object 1 for the Freeman-Davis method (left column) and the proposed method (right column).
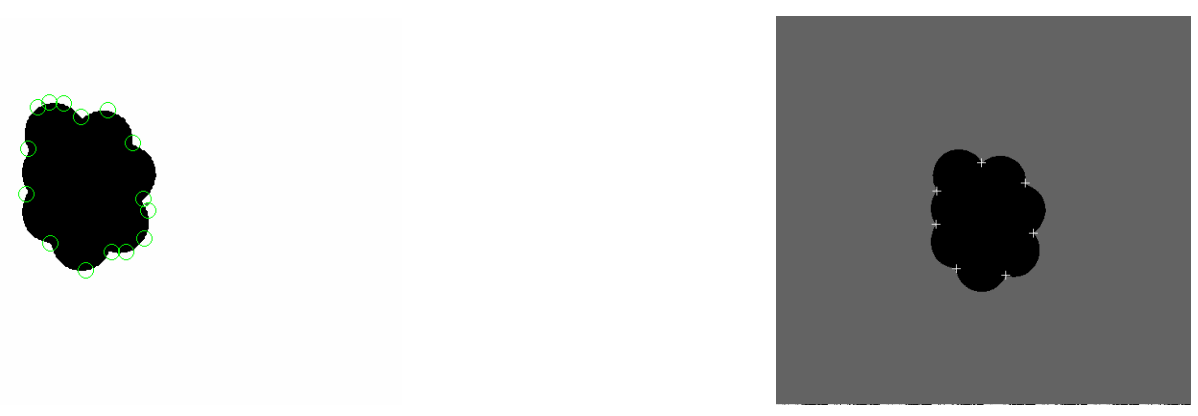

(a) Scaling factor $50 \%$
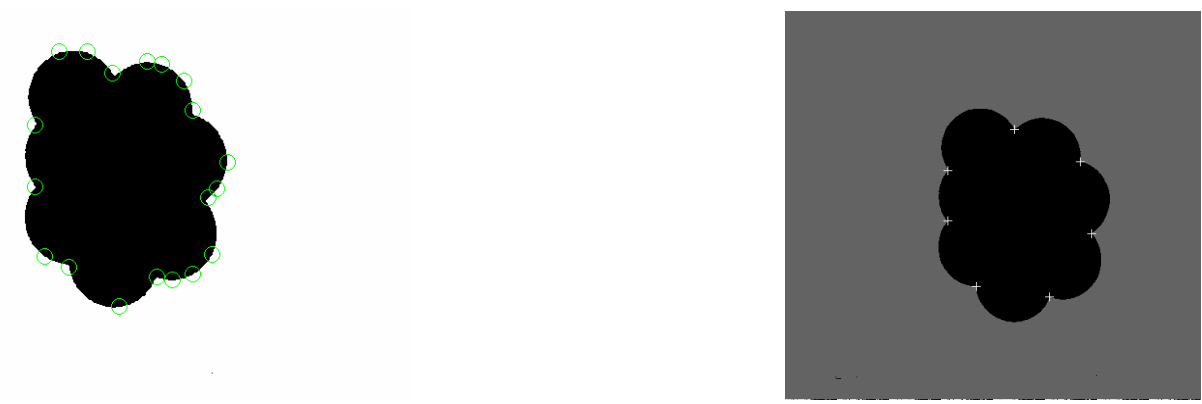

(b) Scaling factor $75 \%$
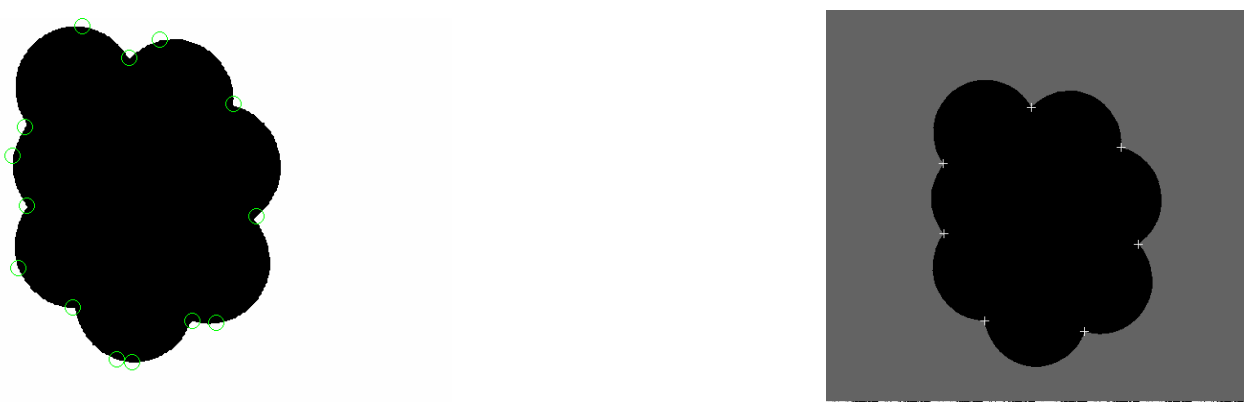

(c) Scaling factor $100 \%$
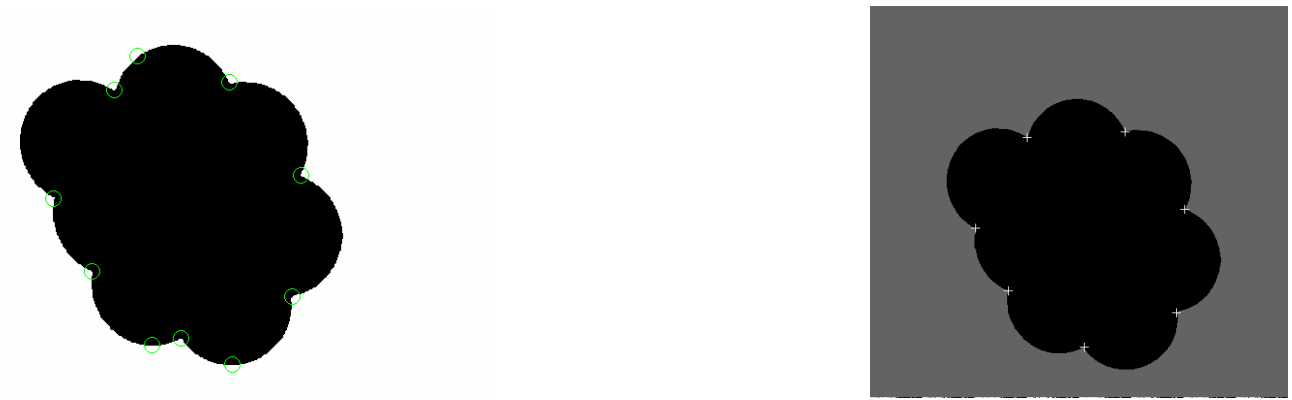

(d) Rotational angle $30^{\circ}$ 

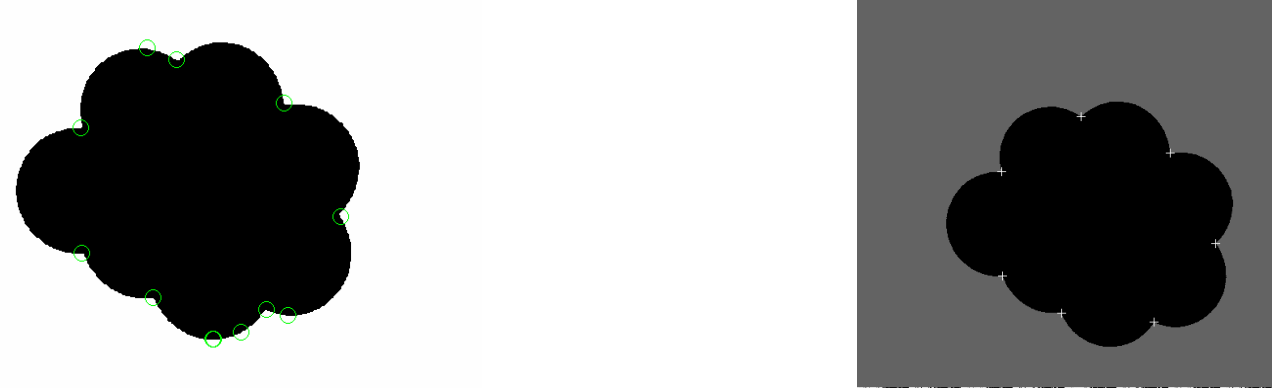

(d) Rotational angle $60^{\circ}$

Figure 4. Detected corners of test object 2 for the Freeman-Davis method (left column) and the proposed method (right column).
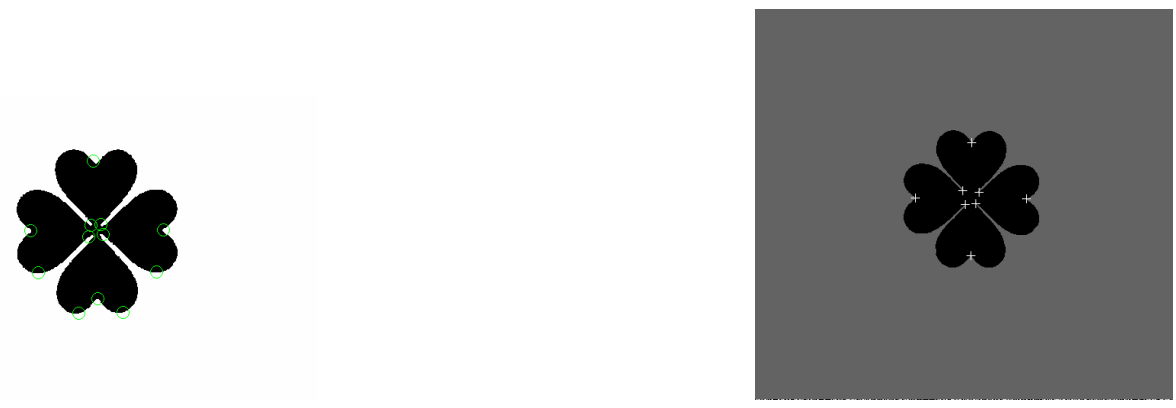

(a) Scaling factor $50 \%$
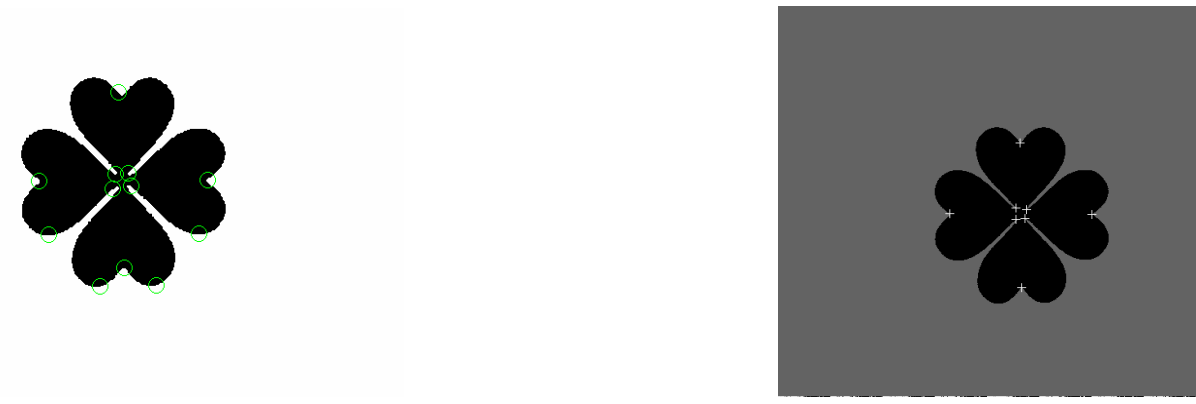

(b) Scaling factor $75 \%$
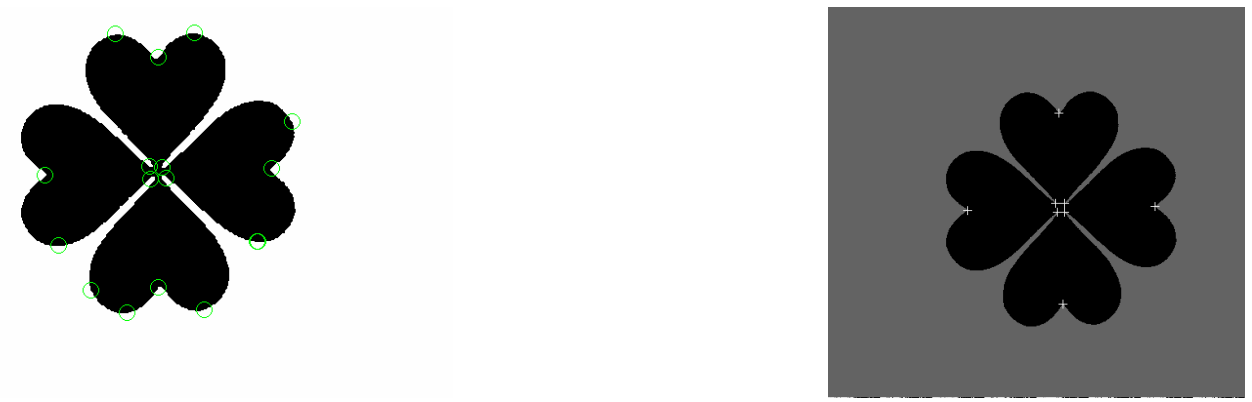

(c) Scaling factor $100 \%$ 

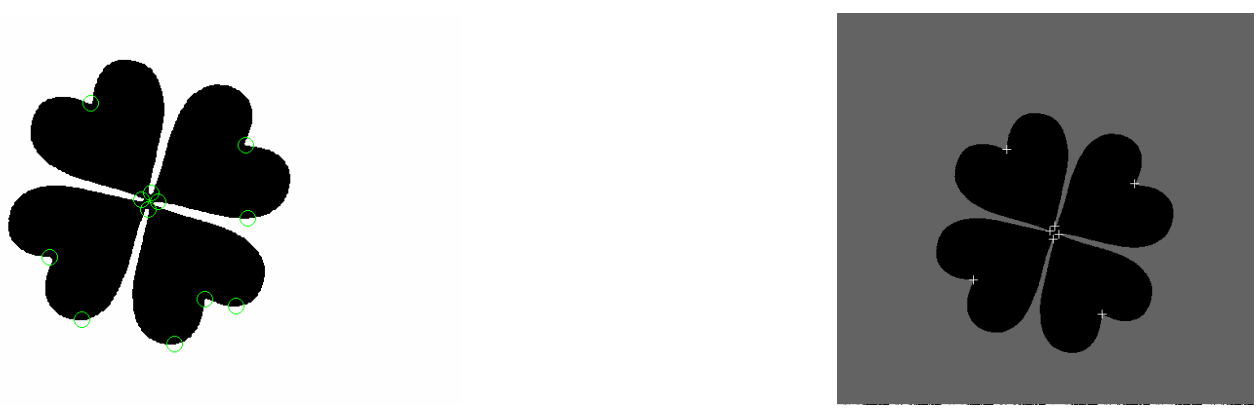

(d) Rotational angle $30^{\circ}$
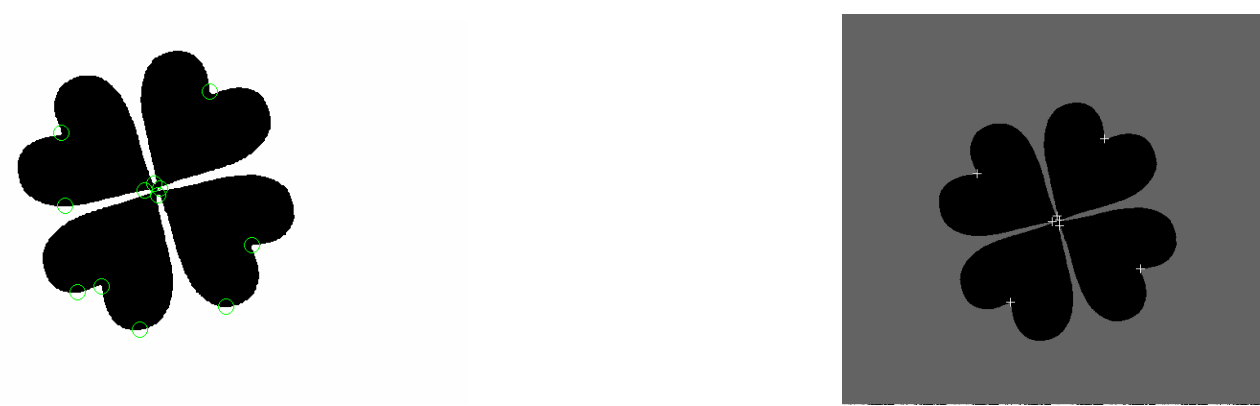

(d) Rotational angle $60^{\circ}$

Figure 5. Detected corners of test object 3 for the Freeman-Davis method (left column) and the proposed method (right column). 